Revista Brasileira de Cartografia

ISSN 1808-0936 | https://doi.org/10.14393/revbrascartogr

Sociedade Brasileira de Cartografia, Geodésia, Fotogrametria e Sensoriamento Remoto

\title{
Desenvolvimento de aplicativo para auxílio à navegação em ambientes internos
}

\section{Development of an app for indoor navigation assistance}

\author{
Carolina Aguilar Aravena ${ }^{1}$ e Luciene Stamato Delazari ${ }^{2}$
}

1 Universidade Federal do Paraná, Programa de Pós-Graduação em Ciências Geodésicas, Curitiba, Brasil. carolina.aguilara@ usach.cl ORCID: https://orcid.org/0000-0002-3284-0288

2 Universidade Federal do Paraná, Programa de Pós-Graduação em Ciências Geodésicas, Curitiba, Brasil. luciene@ufpr.br ORCID: https://orcid.org/0000-0003-0018-085X

Resumo Em ambientes externos o auxílio à navegação do usuário pode ser realizado por meio do uso dos sinais recebidos dos satélites GNSS (Global Navigation Satellite System) ou pelo GSM (Global System for Mobile Communication). No interior das edificações, não é possível utilizar estas tecnologias, o que faz necessário o uso de outros métodos de posicionamento para auxiliar ao usuário. Uma das soluções para o posicionamento neste ambiente é a tecnologia WIFI, que encontra-se implementada em dispositivos móveis, como os smartphones. Entretanto, ainda não existem muitas soluções que podem ser adotadas em ambientes indoor que podem auxiliar no posicionamento do usuário. Nesta pesquisa foi desenvolvido um aplicativo na plataforma Android, com a qual é determinado o posicionamento para o ambiente interno, pelas técnicas do centróide, centróide ponderado e trilateração, para posteriormente ser apresentado em um mapa com a finalidade de assistir na navegação do usuário no ambiente interno. O aplicativo permite ao usuário identificar sua posição no ambiente a partir da interação com o mapa, além de ser apresentado um marcador de posição, indicando o andar e o prédio onde o usuário se encontra. Desta forma auxilia na orientação do usuário, e permite sua localização, além de ser uma ferramenta adequada para este tipo de ambientes. Este trabalho faz parte do projeto UFPR CampusMap da Universidade Federal do Paraná.

Palavras-chave: Android. Posicionamento interno. Redes WIFI.

\begin{abstract}
In outdoor environments, the user's navigation aid can be provided by using signals received from GNSS (Global Navigation Satellite System) satellites or by GSM (Global System for Mobile Communication). Inside the buildings the use of these technologies is not possible, therefore is necessary the use of other positioning methods to assist the user. One of the solutions for positioning in this environment is the WIFI technology, which is implemented in mobile devices such as smartphones. However, there are not yet many solutions that can be adopted in indoor environments that can help the user's positioning. In this research was developed an application on the Android platform, using the techniques of centroid, centroid weighted and trilateration, to provide the positioning for the indoor environment, to be later presented on a map with the purpose of assisting the navigation of the user in the indoor environment. The application allows the user to identify his position in the environment from the interaction with the map. Besides that, is presented a position marker, indicating the floor and building where the user is, which guides the user in a better way, allowing his localization and creating a tool suitable for this type of environment. This research is part of the UFPR CampusMap project of the Federal University of Paraná.
\end{abstract}

Keywords: Android. Indoor positioning. WIFI networks.

\section{INTRODUÇÃO}

A posição de um usuário no espaço pode ser definida de diferentes formas, matematicamente com as coordenadas $\mathrm{x}, \mathrm{y}, \mathrm{z}$ associadas a um referencial global ou local, ou também mediante o uso de feições do ambiente como referência. Ambas as possibilidades podem ser usadas tanto em ambiente externos como internos. Neste contexto, a navegação de um usuário no ambiente interno é baseada em seu conhecimento do local ou com base em pontos de referência. Isto pode ser auxiliado pela utilização de mapas do tipo "You Are Here" (YAH) ou por um sistema que forneça sua posição, já que o usuário deve posicionar-se no ambiente para depois navegar neste (SAROT; DELAZARI, 2018). 
Nos ambientes internos, os elementos utilizados pelos usuários para poder guiar-se podem ser alterados com frequência, e além disso, às vezes é complexo manter a orientação como no espaço externo. Por isso, é necessária a implementação de sistemas de posicionamento neste tipo de ambiente para auxiliar o usuário quando este não conhece o ambiente (ou quando existem mudanças neste) (KANG; LI, 2017).

Quanto aos espaços externos, a solução para o posicionamento através de um smartphone tem sido a utilização do AGPS (Assisted Global Positioning System), que não se pode empregar nos espaços internos devido à atenuação do sinal causada pela estrutura da edificação. O erro de posicionamento gerado pelo AGPS no ambiente interno é aproximadamente 32 metros (FIGUEIREDO; SILVA et al., 2019). Outra possibilidade é a utilização dos serviços baseados na telefonia celular, que não proporcionam a precisão adequada para os ambientes internos, em razão das configurações que estes ambientes apresentam (TREVISAN; PÉREZ, 2017). Neste sentido, Huang et al. (2009) consideram relevante a implementação de um sistema de navegação para estes ambientes, já que múltiplas atividades dos humanos são desenvolvidas nos ambientes internos.

Os Sistemas de Posicionamento Interno (IPS) usam diferentes tecnologias em sua implantação que variam de acordo com a precisão e custo, como as tecnologias por radiofrequências ou por sensores inerciais, entre outras (NAMIOT, 2015). Em relação às radiofrequências, os roteadores WIFI são de uso de grande parte das pessoas e estão normalmente implantados nos prédios, como no caso da Universidade Federal do Paraná, que tem uma rede de roteadores WIFI chamada "UFPR sem fio". Uma das razões deste crescimento é sua integração em dispositivos móveis. Assim, as redes WIFI são uma solução adequada para o posicionamento interno, tendo como características ligações de alta velocidade, uma infraestrutura padronizada e fácil de implantar (KOLODZIEJ; HJELM, 2006; PUERTOLAS; MENDOZA; SANZ, 2013).

Tem-se diversas pesquisas relacionadas ao posicionamento com esta tecnologia como as de Ocaña et. al (2005), Bose e Foh (2007), Honkavirta et al (2009), Avallone e Capdehourat (2010), Gansemer, Großmann e Hakobyan (2010), Llados et. al (2011), Marques, Meneses e Moreira (2012), Kornuta, Acosta e Toloza (2013), Mok e Cheung (2013), Boonsriwai e Apavatjrut (2013), Torres-Sospedra et al. (2014), Conte (2015), Trevisan e Navarro (2017) e Sakr e El-Sheimy (2017). A maioria destas pesquisas realizou estudos relativos ao algoritmo de posicionamento, sem propor a geração de aplicativos que utilizem representações cartográficas.

Neste contexto, esta pesquisa parte da hipótese de que a navegação de um usuário em um ambiente indoor pode ser assistida por meio da utilização de um aplicativo para posicionamento por redes WIFI, se o usuário tiver a capacidade de receber diferentes sinais, em um aplicativo de um dispositivo móvel.

Assim, neste artigo serão apresentadas as diferentes técnicas de posicionamento que podem ser usadas com a tecnologia WIFI, bem como as plataformas com as quais pode ser representado um mapa em um aplicativo móvel. Por fim será apresentado o desenvolvimento de um aplicativo para obter o posicionamento no ambiente interno.

\subsection{Métodos de posicionamento}

Alguns dos métodos que permitem obter a posição mediante a tecnologia WIFI são os seguintes:

\subsubsection{PROXIMIDADE}

A posição do objeto é atribuída ao transmissor mais próximo, portanto, o objeto fica com as coordenadas deste. No caso que sejam dois ou mais transmissores, é considerada a intensidade do sinal mais forte (AL NUAMI; KAMEL, 2011; BROKOVIĆ; SIMIĆ, 2014).

\subsubsection{CENTRÓIDE}

A posição do objeto é a resultante da média das posições dos diferentes transmissores (KOLODZIEJ; HJELM, 2006), com base na formulação apresentada nas Eq. (1) e (2).

$$
\begin{aligned}
& x=\frac{\sum x}{n} \\
& y=\frac{\sum y}{n}
\end{aligned}
$$


onde: $(x, y)$ : coordenadas.

$n$ : número de roteadores.

Além disso, pode-se determinar a posição com os valores ponderados do centróide, no qual são estabelecidos diferentes pesos para os transmissores, de acordo com os valores obtidos de RSSI (Received Signal Strength Indicator - Indicador de força do sinal) ou com a determinação das distâncias de cada um deles (MAUTZ, 2012).

\subsubsection{TRILATERAÇÃO}

A posição de um objeto é determinada medindo a distância em relação a múltiplos pontos de referência. Para duas dimensões são necessários ao menos 3 pontos, enquanto que para três dimensões serão necessários 4 pontos. As coordenadas dos transmissores devem ser previamente conhecidas sendo possível determinar a distância por meio da força do sinal WIFI (GARCÍA, 2008; REGUEIRO, 2014). O objeto detecta a intensidade do sinal dos transmissores, e a posição deste fica determinada pela interseção das direções dos sinais. Com base nos mapas de cobertura circular, estima-se um conjunto de distâncias que são combinadas para identificar pontos de interseção. A área com a maior quantidade de pontos presume-se que corresponde à área do objeto (KOLODZIEJ; HJELM, 2006).

O modelo matemático deste método é o seguinte:

$$
\left(x_{n}-x_{d}\right)^{2}+\left(y_{n}-y_{d}\right)^{2}=r_{n}^{2}
$$

onde: $(x n, y n)$ : coordenadas dos n AP.

$(x d, y d)$ : coordenadas do dispositivo.

$r n$ : distâncias a partir de $\mathrm{n}$ AP ao dispositivo.

$n$ : número de roteadores.

\subsubsection{ANÁLISES DE CENA - FINGERPRINT}

Neste método, observam-se diferentes cenas, as quais são armazenadas, para obter posteriormente a posição de um objeto. Quando existem variações nas cenas observadas, é possível definir se o objeto está em movimento ou não. As cenas podem ser uma imagem, um RSSI, ou qualquer parâmetro físico (GARCÍA, 2008; CONTE, 2015).

Nesta pesquisa são usados os métodos de proximidade, centróide, centróide ponderado e trilateração, devido às limitantes que o método Fingeprint tem para sua utilização em tempo real, já que precisa de uma fase de calibração prévia na qual são armazenados dados de RSSI de diferentes pontos no espaço interno. Além disso, sua precisão está relacionada à densidade de pontos armazenados, e por isso requer um custo computacional maior. Isto limita sua implantação em dispositivos móveis, já que gera um atraso na obtenção da posição de 2 a 3 segundos em uma amostra de 40 observações, o que impossibilita sua aplicação em tempo real.

\subsection{CONTEXTO DE APLICAÇÃO - UFPR CAMPUSMAP}

O UFPR CampusMap (UCM) é um projeto desenvolvido pelo grupo de pesquisa em Cartografia e SIG do Programa de Pós-graduação em Ciências Geodésicas, que tem como objetivo mapear os ambientes externos e internos dos Campus da Universidade Federal do Paraná (UFPR). A ideia surgiu a partir de pesquisas sobre a representação cartográfica dos espaços internos para posteriormente serem adicionadas outras funcionalidades e novos dados, de modo a ter uma representação mais completa destes ambientes. Atualmente, o UCM é apresentado em uma plataforma web onde é possível a visualização da base cartográfica do campus Centro Politécnico (apresentada na Figura 1). 
Figura 1 - Interface da plataforma UFPR CampusMap.

\section{UFPR CampusMap}

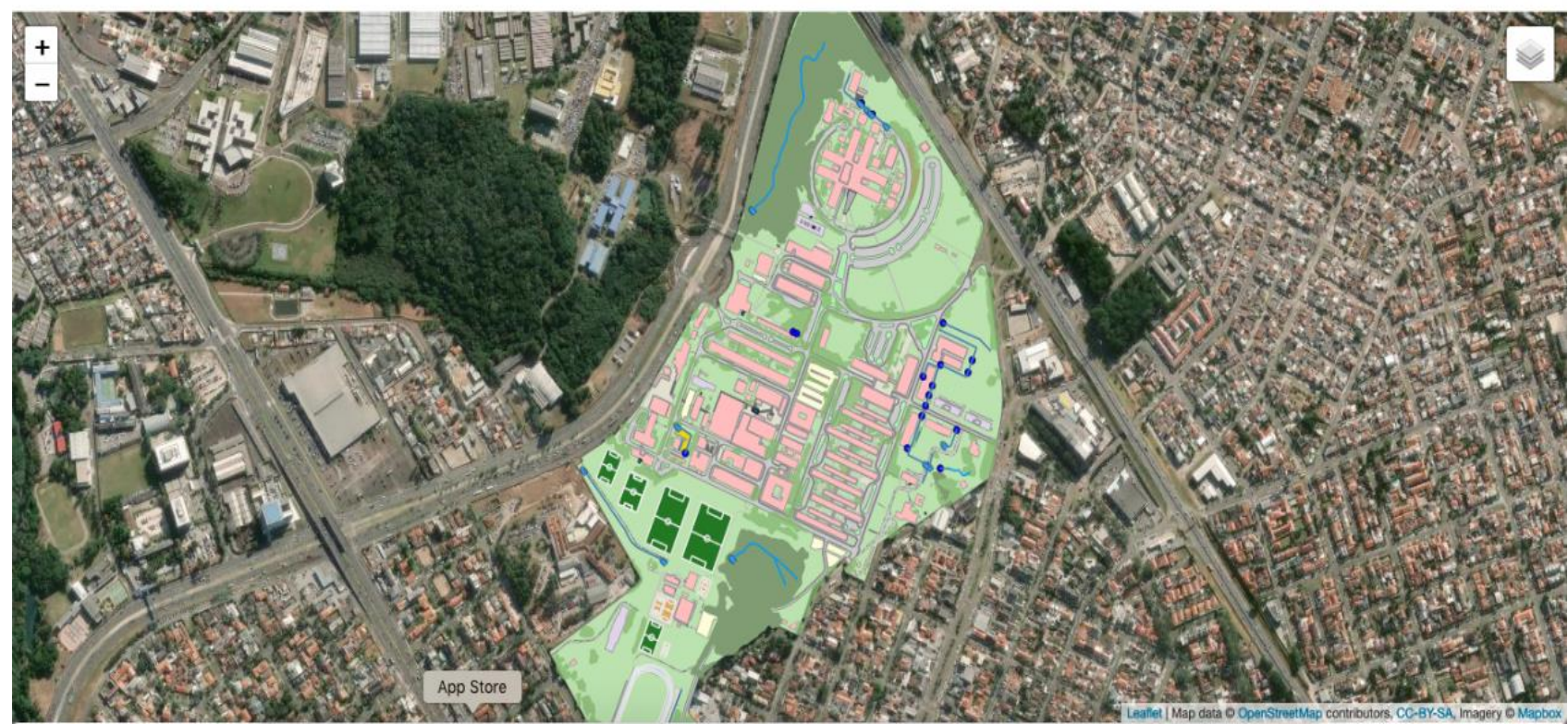

Fonte: UFPR (2019).

Devido à semelhança entre os ambientes que compõem as áreas internas do campus, como corredores e salas, e a alta circulação de pessoas (visitantes e estudantes) é necessária a implementação de um sistema que auxilie o usuário a movimentar-se pelo ambiente.

Antunes (2016) pesquisou a orientação e navegação no ambiente interno através da adoção de etiquetas QR-Code na determinação da posição estimada do usuário em tempo real. Neste trabalho, foi avaliada a obtenção posicional do usuário neste ambiente, e foram estabelecidos pontos de referência utilizados pelo usuário enquanto este realiza o processo de navegação. Concluiu-se que a vinculação do QR-Code ao modelo do banco de dados, auxilia o processo de orientação espacial do usuário. Entretanto, de acordo com DescampsVila et al. (2013) e Regueiro (2014), os marcadores fixos não são considerados sistemas de localização, já que este processo deve ser feito pelo usuário. Ademais, estes sistemas não permitem a navegação, pois não são dinâmicos. Da mesma forma, precisa da ação do usuário, já que ele escolhe quando digitalizar o código.

No caso do desenvolvimento de aplicativos para o posicionamento no ambiente interno, um primeiro trabalho foi feito por Lima (2017). Neste trabalho foi apresentada a base cartográfica da UFPR em um aplicativo desenvolvido para o sistema Android, permitindo o posicionamento com base na leitura de QR Codes. Uma das limitações deste trabalho foi a dificuldade em utilizar a base cartográfica (interior e exterior) por meio de uma conexão com o banco de dados. Deste modo, os dados foram inseridos por meio de arquivos GeoJSON.

\section{DESENVOLVIMENTO}

O desenvolvimento da aplicação foi realizado nas seguintes etapas (Figura 2): 
Figura 2 - Etapas do desenvolvimento do aplicativo.

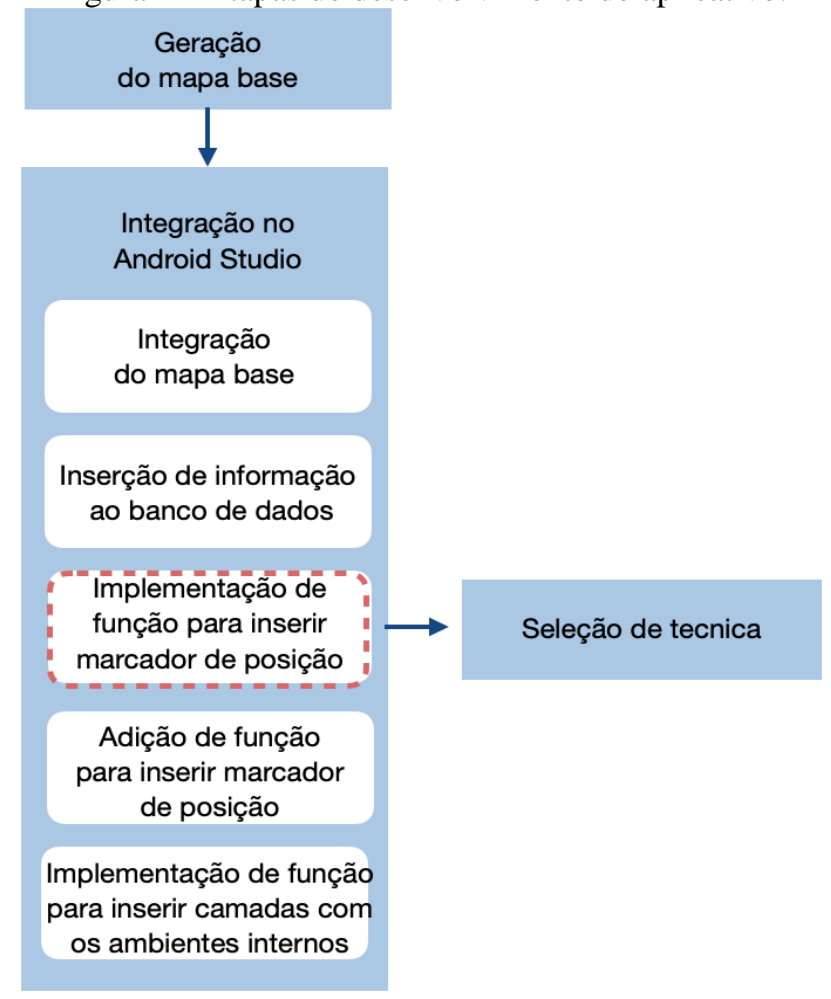

Fonte: Os autores (2021)

\section{1 Área de estudo}

A área de estudo para a realização desta pesquisa (Figura 3) encontra-se localizada no Centro Politécnico, Campus III da Universidade Federal do Paraná, bairro Jardim das Américas, Curitiba, Brasil. Esta é composta por 3 prédios chamados "Bloco didáticos" numerados como IV, V e VI, que contém 2 andares com 194 salas.

Figura 3 - Área de estudo.
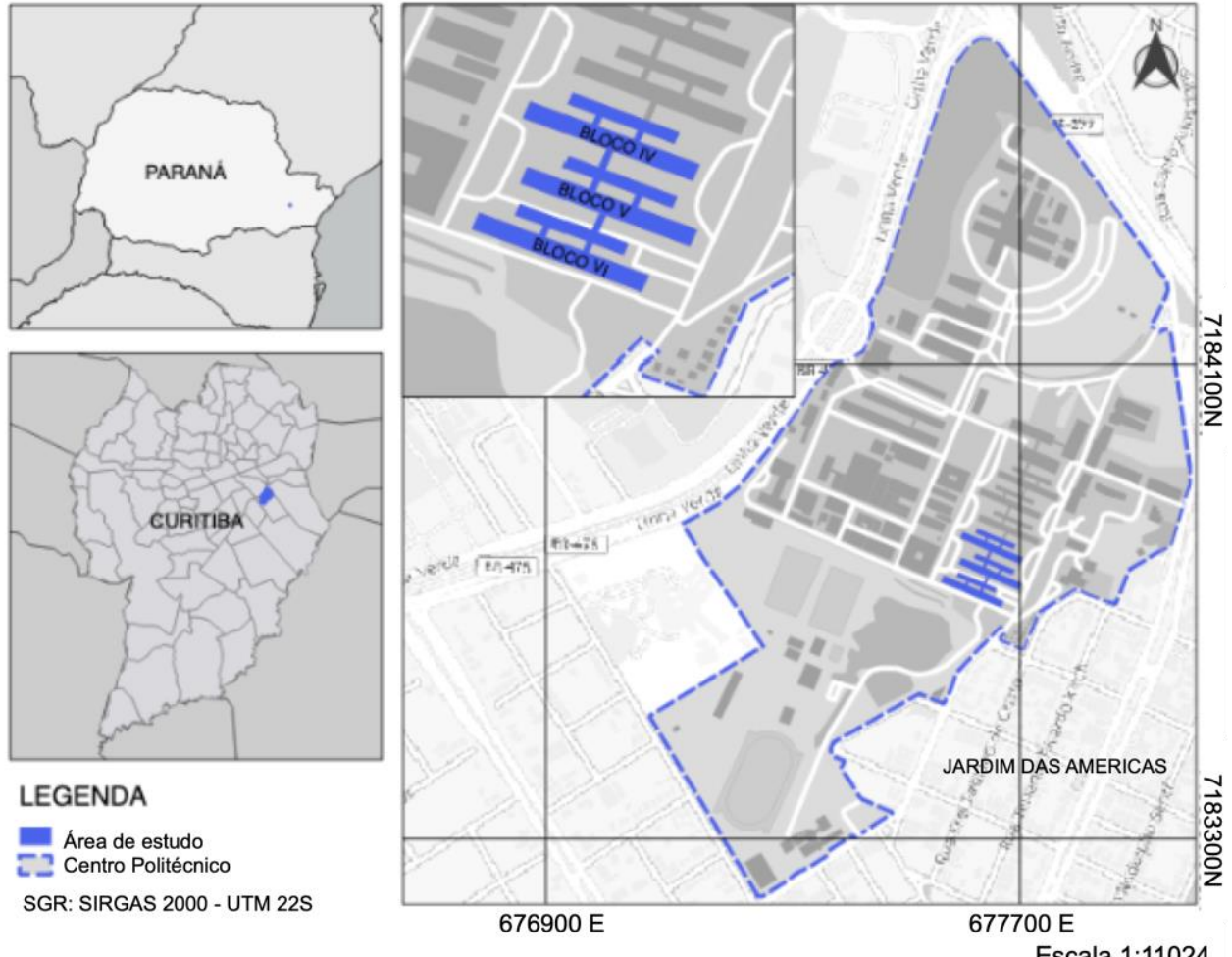

Fonte: Os autores (2021). 


\subsection{Geração do mapa base}

$\mathrm{Na}$ atualidade, a implantação de um mapa "You Are Here" para auxílio no posicionamento indoor é possível mediante diferentes plataformas, como Google Maps, MapQuest, ArcGIS e Mapbox. Nesta pesquisa foi escolhido Mapbox, já que corresponde a um sistema que permite adicionar mapas em diferentes plataformas (web, móvel, dispositivo para navegação veicular e realidade virtual). O Mapbox possui um plugin chamado "Mapbox Android Map" disponível para a plataforma Android que permite adicionar um mapa em um aplicativo para um smartphone com esse sistema operacional. Com este plugin é possível escolher o estilo das cores da base cartográfica, adicionar arquivos GeoJSON, adicionar marcadores, gerar aplicações para navegação exterior com a obtenção da posição por meio do AGPS e das redes móveis (GSM), entre outras funções (MAPBOX, 2019).

Para adicionar a base cartográfica do Campus Centro Politécnico, foi usado o Mapbox Studio, uma plataforma web que permite integrar camadas em diferentes formatos (shapefile, JSON e csv) à base cartográfica do Mapbox, para posteriormente serem usados em mapas para diferentes plataformas. Neste software é possível escolher diferentes estilos de cores de mapa, definir estilos de cores para cada uma das camadas e adicionar manualmente dados. A interface de Mapbox Studio é apresentada na Figura 4.

Figura 4 - Interface de Mapbox Studio.

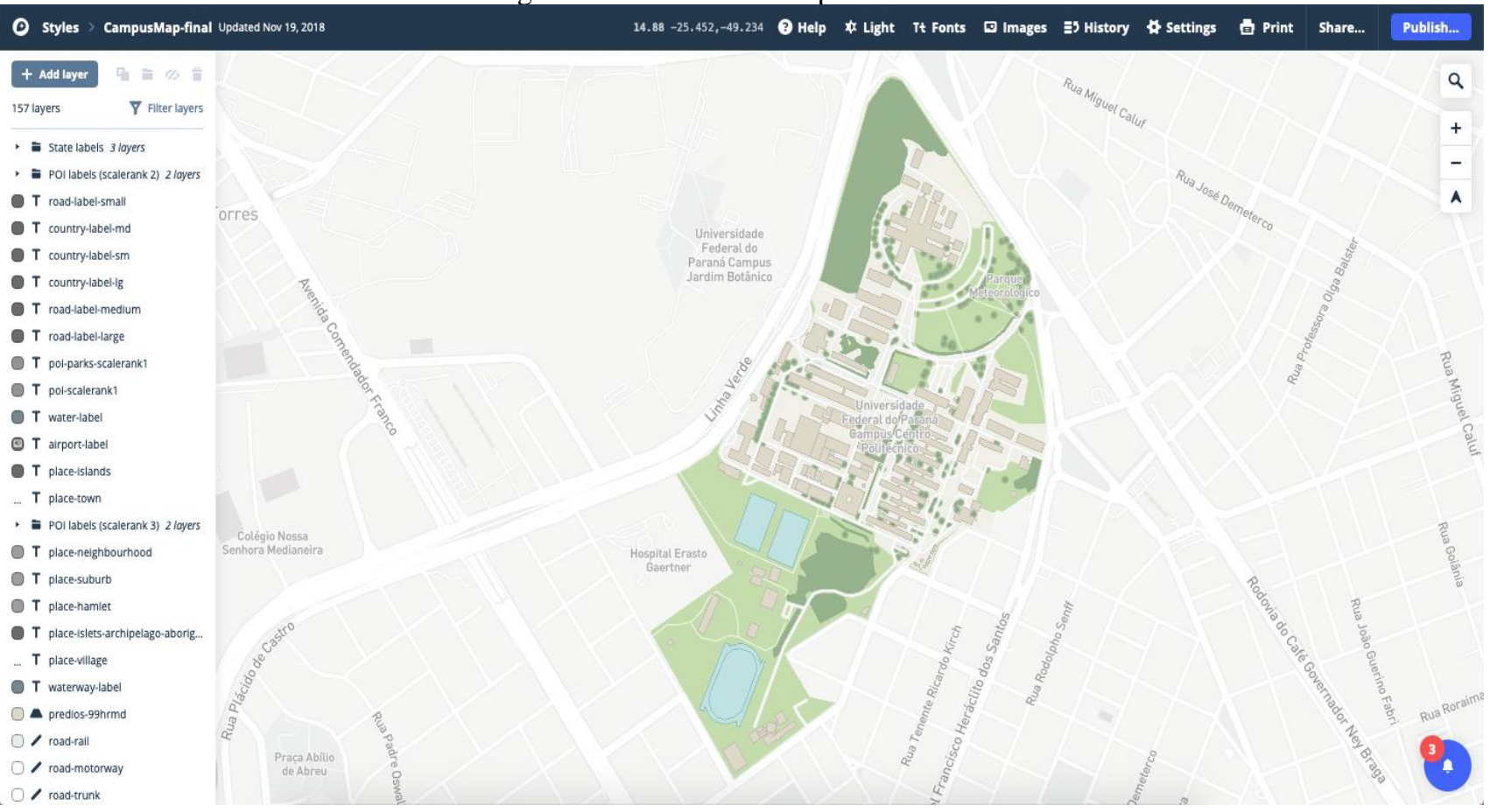

Fonte: Mapbox Studio (2019).

\subsection{Integração no Android Studio}

Com a finalidade de gerar um mapa YAH em tempo real, foram escolhidas as técnicas centróide, centróide ponderado e trilateração, para obter a posição pela tecnologia WIFI. Foi desenvolvido um aplicativo no software "Android Studio" com a linguagem de programação Java, integrando o mapa por meio do Mapbox. O código para realizar este processo é apresentado na Figura 5. 
Figura 5 - Código para gerar o mapa.

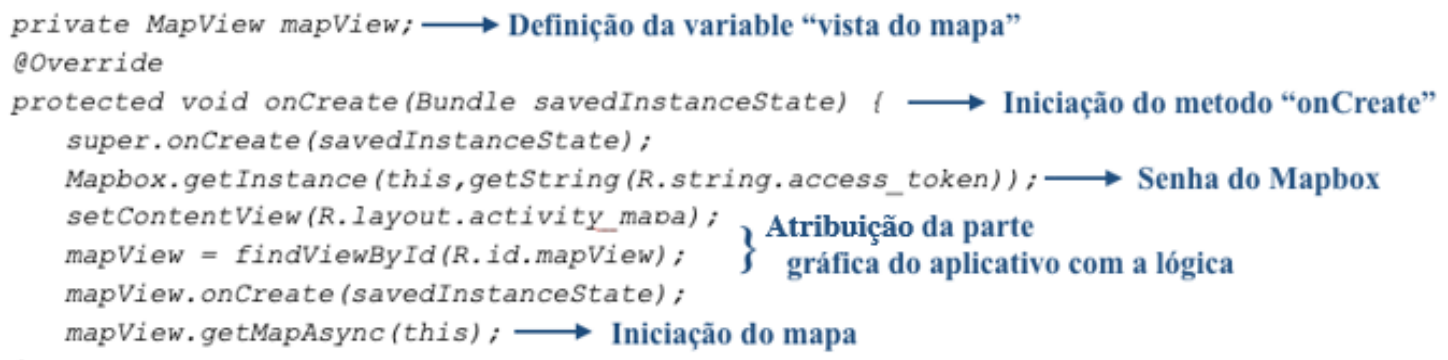

Fonte: Os autores (2021).

Na Figura 5, primeiramente foi definida a variável "mapView", posteriormente foi iniciado o método "onCreate" que cria a interface da aplicação, posteriormente foi inserida a senha do Mapbox (senha fornecida para cada usuário para gerar aplicativos), depois foi feita a conexão da parte gráfica com a parte lógica (código), para finalmente inicializar o mapa.

No desenvolvimento do aplicativo, foram adicionados à base de dados do dispositivo os seguintes dados dos roteadores da área de estudo:

a) SSID (Service Set Identifier): Nome da rede WIFI;

b) BSSID (Basic Service Set Identifier): Identificador único de um roteador WIFI;

c) Andar;

d) Prédio;

e) Coordenadas (Norte e Este): Obtidas a partir da base cartográfica da área de estudo e densificadas a partir das feições internas e medições de distância eletrônica.

Isto foi possível com a utilização do SQlite, que corresponde a uma biblioteca desenvolvida para armazenar dados diretamente em um dispositivo, sem precisar um servidor. Para obter os dados dos roteadores próximos, como o RSSI (Received Signal Strength Indicator) que corresponde à intensidade do sinal e o BSSID para determinar de que roteador correspondia cada RSSI, foi utilizada a biblioteca "WifiInfo".

Mediante estes dados foi possível determinar a posição do smartphone, pelas diferentes técnicas. No caso da técnica do centróide foi determinada a média aritmética das posições dos roteadores que fossem detectados pelo smartphone no momento do posicionamento; no caso da técnica do centróide ponderado, foram inseridas ponderações aos roteadores de acordo com a intensidade do sinal recebido, calculando a média ponderada e finalmente, para a técnica da trilateração de acordo com seu modelo matemático.

$\mathrm{O}$ andar e prédio foram determinados pela técnica de proximidade, na qual o aplicativo estima pela proximidade o roteador mais próximo, consulta no banco de dados e obtém a informação do andar. Em seguida, por meio de um arquivo GeoJSON as posições das salas são apresentadas.

No caso do uso de alíneas, obedecer às seguintes indicações:

\section{RESULTADOS}

Cada técnica foi avaliada em relação à sua acurácia posicional para posicionamento estático e cinemático. O resultado para o posicionamento estático é apresentado na Figura 6. Os deslocamentos no caso da técnica centróide foram entre 0,52 e 18,69 metros; para o centróide ponderado estes se encontram entre 0,42 e 19,15 metros; e no caso da trilateração estiveram entre 4,68 e 13,79 metros. Na Tabela 1 é apresentada a discrepância média e o desvio padrão para cada técnica. 
Tabela 1-Resultados Posicionamento estático.

\begin{tabular}{l|c|c}
\hline \multicolumn{1}{c|}{ Técnica } & $\begin{array}{c}\text { Discrepâncias planimétrica média } \\
(\mathbf{m})\end{array}$ & Desvio padrão (m) \\
\hline Centróide & 10,43 & $\pm 6,06$ \\
Centróide ponderado & 7,39 & $\pm 4,39$ \\
Trilateração & 8,38 & $\pm 3,14$ \\
\hline
\end{tabular}

Fonte: Os autores (2021).

Figura 6 - Resultado para o posicionamento estático.
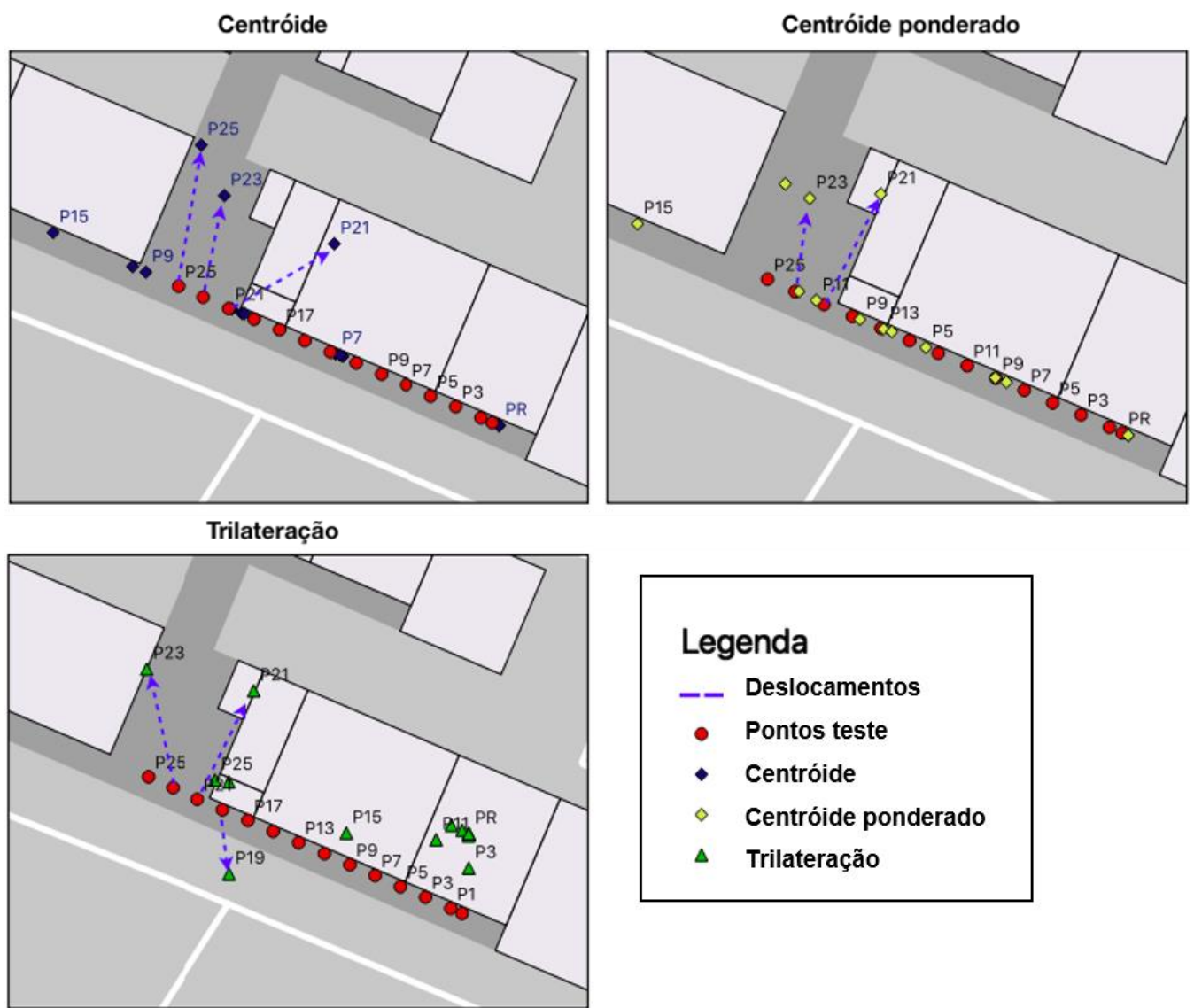

Fonte: Os autores (2021).

No caso do posicionamento cinemático, os resultados para o mesmo trajeto são apresentados na Figura 7, onde o trajeto resultante com a técnica do centróide e o centróide ponderado tiveram um comportamento muito similar, sendo os mais representativos de acordo com o trajeto original comparado com a técnica da trilateração. 
Figura 7 - Resultados para o posicionamento cinemático.

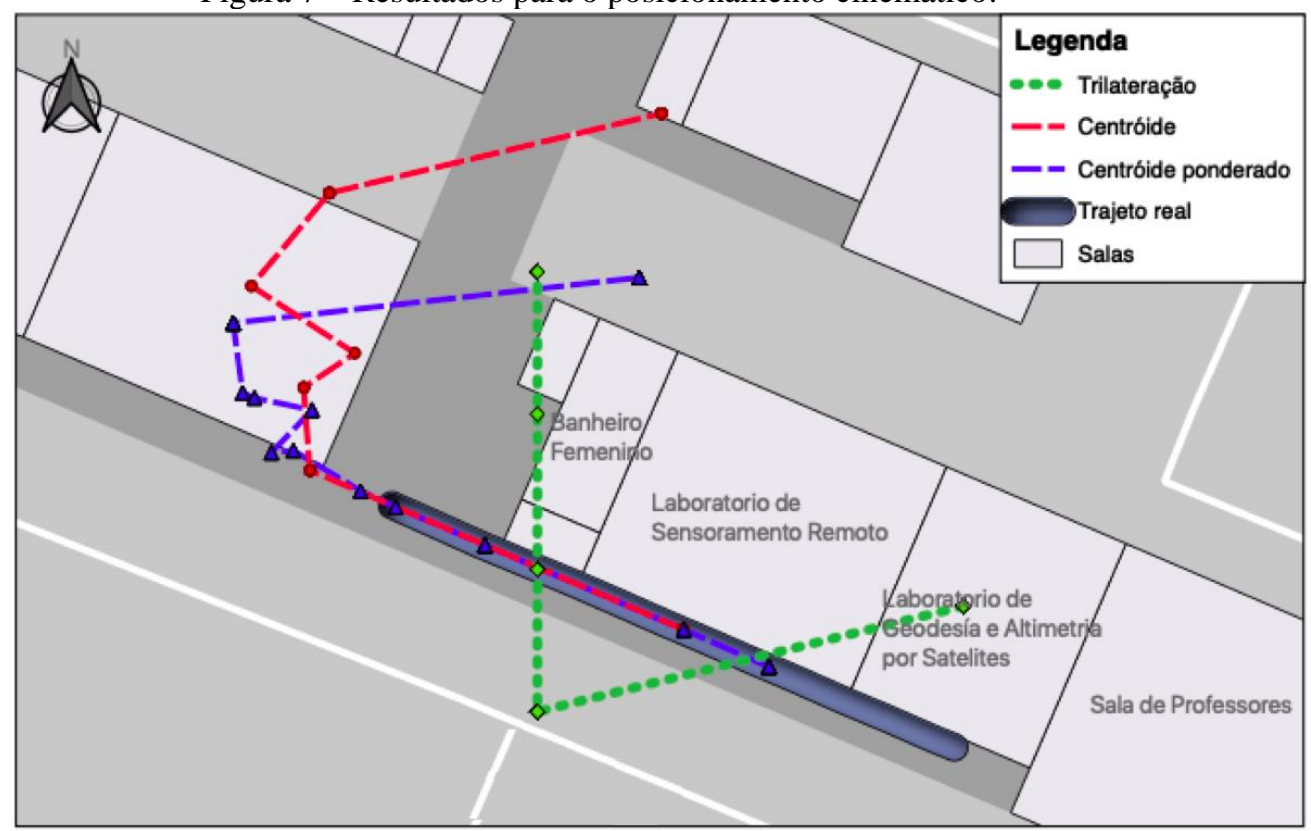

Fonte: Os autores (2021).

Como a técnica do centróide ponderado manteve a menor discrepância em comparação ao trajeto real, o resultado da posição obtida por esta técnica foi associado ao marcador no mapa. Com a finalidade de melhorar o auxílio à localização do usuário, foi fornecido o número do andar e prédio (na parte superior da tela) obtidos pela técnica de proximidade. A interface resultante é apresentada na Figura 8.

Figura 8 - Interface do aplicativo.

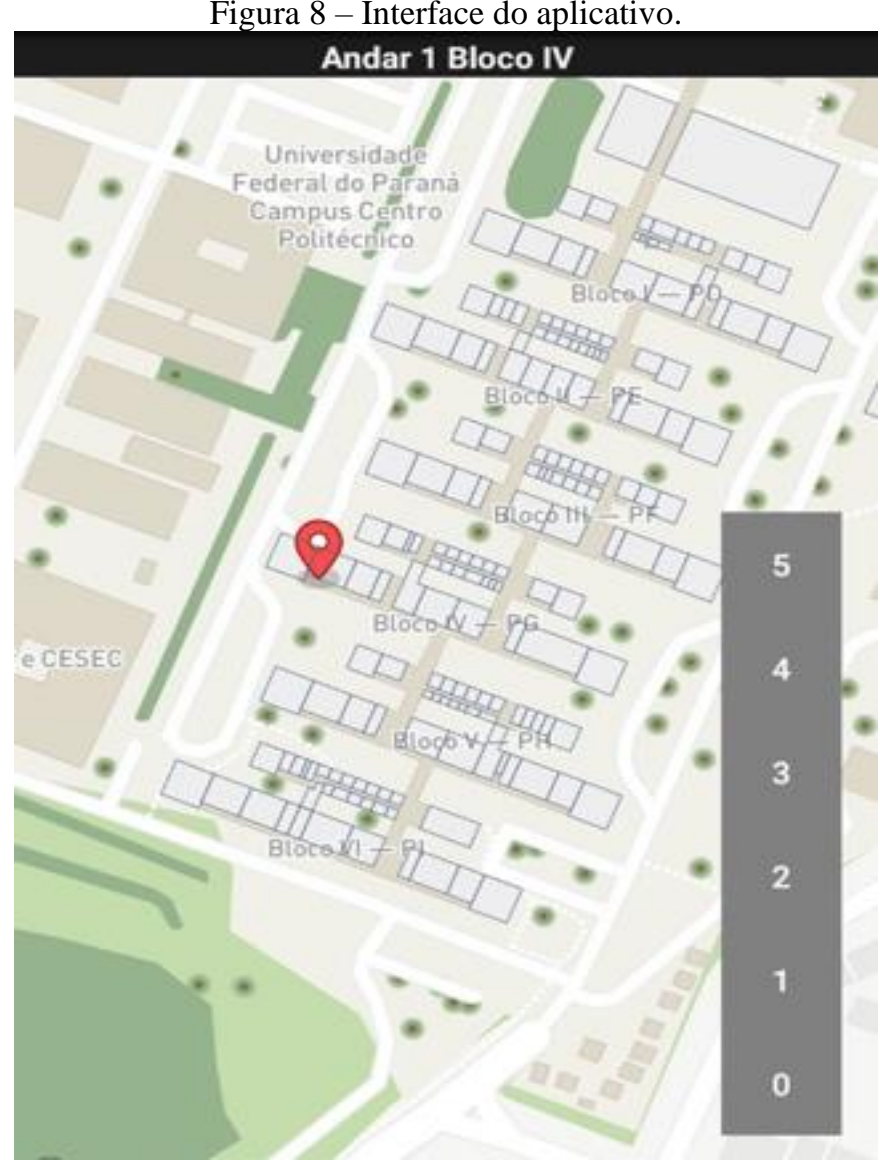

Fonte: Os autores (2021). 


\section{CONCLUSÕES}

O uso de plataformas web para o desenvolvimento de mapas permite a sua integração em navegadores ou em dispositivos móveis. Como foi apresentado neste trabalho a plataforma Mapbox foi usada para gerar um mapa com o objetivo de obter a visualização da base cartográfica do projeto UFPR CampusMap em dispositivos móveis Android e assim, auxiliar a navegação do usuário no ambiente interior, a qual é obtida a partir de posicionamento pela tecnologia de redes WIFI.

A plataforma Mapbox fornece múltiplas funcionalidades que permitem gerar uma base cartográfica com informação própria, permitindo editá-la no sistema, para finalmente ser utilizada em qualquer aplicativo. Além disso, integrando com a inserção de posição mediante a tecnologia WIFI, permite que o usuário possa localizar-se no ambiente, fornecendo uma ferramenta adequada para auxiliar o usuário nos ambientes interiores.

Como trabalhos futuros, é necessária a realização de testes com usuários para aumentar a usabilidade desta plataforma, como também seria importante inserir a simbologia das diferentes feições presentes no ambiente, com a finalidade de auxiliar o entendimento do ambiente.

Para a melhoria da qualidade do resultado do posicionamento, devem ser desenvolvidas técnicas de filtragem e modelagem dos dados.

\section{Agradecimentos}

A autora Carolina Aguilar Aravena agradece à Organização dos Estados Americanos (OEA) e ao Grupo Coimbra das Universidades Brasileiras (GCUB), pela bolsa de estudos de mestrado. A autora Luciene Stamato Delazari agradece ao Conselho Nacional de Desenvolvimento Científico e Tecnológico pela concessão da Bolsa Produtividade em Pesquisa (CNPq -Processo nº 310312 / 2017-5).

\section{Contribuição dos Autores}

O A primeira autora desenvolveu a curadoria dos dados com o aval da segundo autora. Ambas as autoras participaram durante o resto das etapas da pesquisa como: conceptualização, metodologia, validação, redação, revisão e edição.

\section{Conflitos de Interesse}

Os autores declaram que não há conflitos de interesse.

\section{Referências}

AL NUAMI, K. KAMEL, H. A Survey of Indoor Positioning Systems and Algorithms. In: International Conference on Innovations in Information Technology, 2011. Anais... Abu Dhabi, p. 185-190, 2011.

ANTUNES, A. P. Avaliação de pontos de referência com uso de Qr-Code para posicionamento em ambiente indoor. 96 f. Dissertação (Mestrado em Ciências Geodésicas) - Programa de Pós-Graduação em Ciências Geodésicas, Universidade Federal do Paraná, Cuitiba, 2016.

AVAllone, C; CAPDEHOURAT, G. Posicionamiento indoor con señales de WIFI. $18 \mathrm{f}$. Trabalho (Graduação Engenharia Elétrica) - Departamento de Ingeniería Eléctrica. Universidad de la República de Uruguay, Montevideo, 2010.

BOSE, A; FOH, C. A Practical Path Loss Model For Indoor WiFi Positioning Enhancement. In: International Conference on Information, Communications and Signal Processing (ICICS). Singapore, Sixth, 2007. Anais... p.1-5 2007.

BOONSRIWAI, S; APAVATJRUT, A. Indoor WIFI localization on mobile devices. In: International Conference on Electrical Engineering / Electronics, Computer, Telecommunications and Information 
Technology, 10th, 2013. Anais... Krabi, p. 1-5, 2013.

CONTE, E. Algoritmos de análise de cena para localização indoor via redes IEEE 802.11. 96 f. Dissertação (Mestrado em Engenharia de Automação e Sistemas) - Programa de Pós-graduação em Engenharia de Automação e Sistemas, Universidade Federal de Santa Catarina, Florianópolis, 2015.

DESCAMPS-VILA, L. PÉREZ, A. CONESA, J. Integración de un sistema de posicionamiento Indoor en aplicaciones SIG para dispositivo movil. In: Jornada de SIG libre, VII, 2013. Anais... Girona, 2013.

DELAZARI, L. ERCOLIN FILHO, L. VIANA SAROT, R. FARIAS, P. P. ANTUNES, A. BARBOSA DOS SANTOS, S. 9 - Mapping Indoor Environments: Challenges Related to the Cartographic Representation and Routes. In CONESA, J. (ed.) PÉREZ- NAVARRO, A. (ed.) TORRES-SOSPEDRA, J. MONTOLIU, $\mathrm{R}$ (ed.). Geographical and Fingerprinting Data to Create Systems for Indoor Positioning and Indoor/Outdoor Navigation. Academic Press. 2019, p. 169-186.

FIGUEIREDO E SILVA, P. RICHTER, P; TALVITIE, J. LAITINEN, E. LOHAN, E.S. 13 - Challenges and Solutions in Received Signal Strength-Based Seamless Positioning. In: CONESA, J. (ed.) PÉREZNAVARRO, A. (ed.) TORRES-SOSPEDRA, J. MONTOLIU, R (ed.). Geographical and Fingerprinting Data to Create Systems for Indoor Positioning and Indoor/Outdoor Navigation. Academic Press. 2019, p. 249-285.

GARCÍA, E. Técnicas de Localización en Redes Inalámbricas de Sensores. 22 f. Trabalho (Graduação Engenharia Informática) - Instituto de Investigación en Informática de Albacete, Departamento de Sistemas Informáticos, Universidad de Castilla-La Mancha, 2008.

GANSEMER, S; GROßMANN, U; HAKOBYAN, S. RSSI-based Euclidean Distance algorithm for indoor positioning adapted for the use in dynamically changing WLAN environments and multi-level buildings. In: 2010 International Conference on Indoor Positioning and Indoor Navigation, Anais... p. 1-6, Zurich, 2010.

HONKAVIRTA, V; PERÄLÄ, T; ALI-LÖYTTY, S; PICHÉ, R. A comparative survey of WLAN location fingerprinting methods. In: Workshop on Positioning, Navigation and Communication, 6th, 2009. Anais... p. $243-251,2009$.

HUANG, H. GARTNER, G. LI, Y. SCHMIDT, M. Smart Environment for Ubiquitous Indoor Navigation. In: International Conference on New Trends in Information and Service Science (NISS 2009), Anais... Beijing, p. 176-180, 2009

KANG, H. K. LI, K. J. A standard indoor spatial data model-OGC IndoorGML and implementation approaches. ISPRS International Journal of Geo-Information, 6(4), p. 116, 2017. DOI: 10.3390/ijgi6040116

KOLODZIEJ, K. HJELM, J. Local Positioning Systems. CRC Press, Taylor e Francis Group, 2006.

KORNUTA, C; ACOSTA, N; TOLOZA, J. Posicionamiento WIFI con variaciones de fingerprint. In: Congreso Argentino de Ciencias de la Computación, XVIII, 2013. Anais... Argentina, 2013.

LIMA, C. Desenvolvimento de aplicativo para dispositivos móveis com mapas indoor para o projeto UFPR Campus map. 36 f. Trabalho (Graduação Engenharia Cartográfica e de Agrimensura), Universidade Federal do Paraná, 2017.

LLADÓS, J; PALLEJÀ, T; TRESANCHEZ, M; TEIXIDÓ, M; FONT, D; PALACÍN, J. Experiencia de auto localización en un recinto universitario a partir de la red WiFi existente. In: Seminario Anual de Automática, Electrónica Industrial e Instrumentación Badajoz, 2011. Anais... España, p. 481-484. 2011

MAPBOX. Maps SDK for Android. Disponivel. Site <https://docs.mapbox.com/android/maps/overview/> Acesso em: 12 abr. 2019.

MARQUES, N; MENESES, F; MOREIRA, A. Combining similarity functions and majority rules for multibuilding, multi-floor, WIFI positioning. In: Indoor Positioning and Indoor Navigation (IPIN), 2012. Anais... Sidney, Austrália, 2012.

MAUTZ, R. Indoor Positioning Technologies. 128 f. Dissertação (Mestrado Positioning and Engineering Geodesy), Deparment of Civil, Institute of Geodey and Photogrametry, Enviromental and Geomatic 
Engineering. Zurich, 2012.

MOK, E.; CHEUNG, B.K. An Improved Neural Network Training Algorithm for Wi-Fi Fingerprinting Positioning. ISPRS International Journal of Geo-Information, v. 2, i. 3, p. 854-868, 2013. DOI: 10.3390/ijgi2030854

NAMIOT, D. On Indoor Positioning. International Journal of Open Information Technologies, v. 3, n. 3, 2015.

OCAÑA, M; BERGASA, L. M; SOTELO, M. A; NUEVO, J; FLORES, R. Indoor Robot Localization System Using WiFi Signal Measure and Minimizing Calibration Effort. In: IEEE International Symposium on Industrial Electronics, 2005. Anais... Dubrovnik, Croatia, p. 1545-1550, 2005.

PUERTOLAS, J. A. MENDOZA, A. SANZ, I. Smart Indoor Positioning/Location and Navigation: A Lightweight Approach. International Journal of Interactive Multimedia and Artificial Intelligence, v.2, n. 2, 2013 DOI: 10.9781/ijimai.2013.225

REGUEIRO, C. Error en el posicionamiento Indoor en dispositivos móviles. 23f. Dissertação (Mestrado Software Livre) - Universitat Oberta de Catalunya, Barcelona, 2014.

SAROT, R.V. DELAZARI, L.S. Evaluation of mobile device indoor maps for orientation tasks. Boletim de Ciências Geodésicas, v. 24, n. 4, p. 564-584, 2018. DOI:10.1590/s1982-21702018000400034

SAKR, M; EL-SHEIMY, N, Efficient Wi-Fi signal strength maps using sparse Gaussian process models. In: 2017 International Conference on Indoor Positioning and Indoor Navigation (IPIN), 2017. Anais... Sapporo, p. 1-8, 2017.

TORRES-SOSPEDRA, J; MONTOLIU, R; MARTÍNEZ-USÓ, A; AVARIENTO, J; ARNAU, T; BENEDITO-BORDONAU, M; HUERTA, J. UJIIndoorLoc: A new multi-building and multi-floor database for WLAN fingerprint-based indoor localization problems. In: 2014 International Conference on Indoor Positioning and Indoor Navigation (IPIN), 2014. Anais... Busan, p. 261-270, 2014.

TREVISAN, D. PÉREZ, A. Influencia de la presencia de personas en sistemas de posicionamiento indoor mediante Wi-Fi fingerprinting.38 f. Trabalho (Graduação Engenharia em informática) - Universitat Oberta de Catalunya, Espanha, 2017.

UFPR. Universidade Federal do Paraná. UFPR CampusMap. Site <http://www.campusmap.ufpr.br/> Acesso: 11 abr. 2019.

\section{Biografia do autor principal}

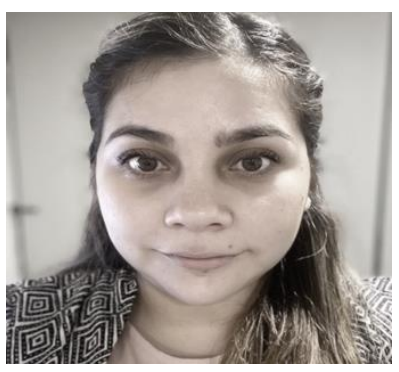

Carolina Aguilar Aravena, nasceu no Chile em 1991. Engenheira em Geomensura pela Universidade de Santiago do Chile (USACH) em Santiago - Chile (2014). Mestre em Ciências Geodésicas pelo Programa de Pós-Graduação em Ciências Geodésicas (PPGCG) da UFPR (2019). Bolsista de mestrado pela Organização dos Estados Americanos (OEA). Atualmente encontra-se trabalhando como Consultora GIS na Superintendência do Meio Ambiente no Chile. Atua nas áreas: Sistemas de informação Geografica, IDE, Banco de dados e Web GIS. 